\title{
Gene frequency clines at X-linked or haplodiploid loci
}

\author{
Robin E. Owen
}

Department of Biology, University of Calgary, Calgary, Alberta, Canada, T2N 1 N4.

The equations governing gene frequency clines at $\mathrm{X}$-linked or haplodiploid loci are derived. Clines at X-linked loci will be identical to corresponding clines at autosomal loci only under three restrictive conditions (a) no dominance, (b) dispersal distance the same in each sex, (c) average effect of alleles same in each sex. If any of these conditions are not met then the males and females will have different gene frequencies. Clinal variation in colour morph frequency in the bumble bee Bombus melanopygusis analysed. It is concluded that a minimum selection intensity acting on the queens on the order of 1 per cent or less is sufficient to maintain the cline.

\section{INTRODUCTION}

Gene frequency clines (gradual changes in gene frequency with distance) occur in many populations of plants and animals. The existence of a cline is often interpreted as evidence of spatial variation in selection on the alleles involved, whether the cline is formed in situ or results from secondary intergradation of previously differentiated populations (Endler, 1977). The shape and other characteristics of clines resulting from selection depend mainly on the intensity of the selection, the level of gene flow, the degree of dominance and the presence or absence of heterozygote advantage or disadvantage (Slatkin, 1973; May et al., 1975).

Cline theory for diploid or autosomal loci has been developed for two types of selection regime. (a) Step or ecotone clines; in this model the selective advantage of the alleles is assumed to reverse abruptly at a boundary. Solutions for clines in an infinite environment (i.e., where the selection regime extends infinitely in both directions) are given by Slatkin (1973), May et al. (1975) and Nagylaki (1976) and for the case of complete dominance by Haldane (1948). Two-locus clines are analysed by Slatkin (1975). Solutions for step clines in a finite environment are given by Nagylaki (1975, 1976) and Fleming (1975). (b) Gradient clines; in this model selection is assumed to change gradually along a gradient. A numerical solution is given by Fisher (1950) and properties of gradient clines are discussed by Slatkin (1973), May et al. (1975) and Endler (1977).

In this paper the basic equations governing gene frequency clines at X-linked or haplodiploid loci are derived. This case has not been previously analysed, possibly because few clines in haplodiploids have been described though some are known (e.g., Johnson et al., 1969; Owen and Plowright, 1980). The model for a step cline in an infinite environment is analysed in some detail. It is shown that some quite restrictive conditions (no dominance, equal dispersal by each sex, average effect of alleles same in each sex) are required for clines in haplodiploids to be identical to those in diploids. Some general properties of haplodiploid clines are derived by examining the limiting case of no dispersal by males, which allows the equations to be put in a form to which there is an analytical solution.

Finally, the cline in colour morph frequency in the bumble bee Bombus melanopygus Nylander is analysed in the light of the theory developed, in order to obtain a rough estimate of the selection intensity requried to maintain the cline.

\section{CLINES AT X-LINKED OR HAPLODIPLOID LOCI}

In this section a model for clines at haplodiploid loci is developed and a diffusion approximation analogous to that for diploid loci is derived. 
The following assumptions are necessary, some of which are given by Slatkin (1973):

(a) The population is continuously distributed in a region infinite in length.

(b) The effects of genetic drift are ignored, but on account of haplodiploidy the gene frequencies will not necessarily be in Hardy-Weinberg equilibrium after mating.

(c) Generations are discrete and non-overlapping.

(d) In each generation females and males mate and offspring are produced upon which natural selection acts. Dispersal, followed by mating to start the next generation, then occurs.

(e) Mating and selection occur independently at each location before the offspring disperse.

A model for a cline must combine a term for selection at each location with a term for dispersal (Roughgarden, 1979). Two gene frequency equations, one for females and one for males, are required for haplodiploid loci because selection and dispersal may not necessarily be the same for each sex. The following derivation uses an approach similar to that of Roughgarden's treatment of the diploid case (1979).

Consider a single locus with two alleles $A_{1}$ and $A_{2}$ at frequencies $p, q$ in females and $P, Q$ in males respectively, and genotypic viabilities,

$$
\text { Females, } \begin{array}{rr}
A_{1} A_{1}: w_{11} & \text { Males, } A_{1}: v_{1} \\
A_{1} A_{2}: w_{12} & \\
A_{2} A_{2}: w_{22} & A_{2}: v_{2}
\end{array}
$$

Let $M_{f}(x-y)$ be the probability that an individual female disperses from location $y$ to location $x$, then the gene frequency at location $x$ in generation $t+1$

$$
\begin{aligned}
& P_{t+1}(x)=\int_{-\infty}^{\infty} M_{f}(x-y)
\end{aligned}
$$

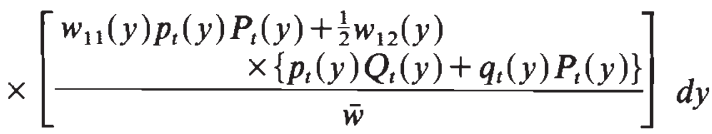

where

$$
\begin{aligned}
\bar{w}= & w_{11}(y) p_{t}(y) P_{t}(y)+w_{12}(y) \\
& \times\left\{p_{t}(y) Q_{t}(y)+q_{t}(y) P_{t}(y)\right\} \\
& +w_{22}(y) q_{t}(y) Q_{t}(y) .
\end{aligned}
$$

Similarly for males

$$
P_{t+1}(x)=\int_{-\infty}^{\infty} M_{m}(x-y)\left[\frac{v_{1}(y) p_{t}(y)}{\bar{v}}\right] d y
$$

where $\bar{v}=v_{1}(y) p_{t}(y)+v_{2}(y) q_{t}(y)$.
These integral equations cannot be solved directly. To make some progress a diffusion equation that approximates the original model must be derived. Time is now considered to be continuous rather than discrete, therefore the total change in gene frequency at location $x=$ change in gene frequency due to selection + change in gene frequency due to dispersal (Roughgarden, 1979).

Consider selection first. The equations for gene frequency change in haplodiploids with weak selection (i.e., $\bar{w} \cong 1, \bar{v} \cong 1$ ), given by Owen (1980), are

$$
\Delta p=p q \alpha-\frac{1}{2}(p-P)
$$

and

$$
\Delta P=p q \beta+(p-P)
$$

where $\alpha=\left(w_{11}-w_{12}\right) p+\left(w_{12}-w_{22}\right) q$ and

$$
\beta=v_{1}-v_{2} \text {. }
$$

These equations (3) and (4), although approximate, are in fact remarkably accurate even with moderately strong selection as is shown in the Appendix.

Let the selective values take the form

$$
\begin{array}{ll}
w_{11}=1+s_{f}(x) & v_{1}=1+s_{m}(x) \\
w_{12}=1+h s_{f}(x) & \\
w_{22}=1-s_{f}(x) & v_{2}=1-s_{m}(x)
\end{array}
$$

where $s_{f}(x)$ and $s_{m}(x)$ are the selection coefficients on the females and males respectively at location $x$ and $h$ is a measure of dominance. Equations (3) and (4) then become

$$
\begin{aligned}
\Delta p= & s_{f}(x) p(x, t) q(x, t) \\
& \times\{1+h[1-2 p(x, t)]\} \\
& -\frac{1}{2}[p(x, t)-P(x, t)] \\
\Delta P= & 2 s_{m}(x) p(x, t) q(x, t) \\
& +[p(x, t)-P(x, t)] .
\end{aligned}
$$

Now consider dispersal. The standard deviations $1_{f}$ and $1_{m}$ of $M_{f}$ and $M_{m}$ respectively, are measures of the average dispersal distance. Therefore the approximate change in gene frequency (Roughgarden, 1979), for females is given by

and for males by

$$
\frac{1_{f}^{2}}{2} \frac{\partial^{2}}{\partial x^{2}} p(x, t)
$$

$$
\frac{1_{m}^{2}}{2} \frac{\partial^{2}}{\partial x^{2}} P(x, t)
$$


Combining the selection and dispersal components yields two differential equations, one for $p(x, t)$ and the other for $P(x, t)$,

$$
\begin{aligned}
& \frac{\partial p(x, t)}{\partial t}= \frac{1_{f}^{2}}{2} \frac{\partial^{2}}{\partial x^{2}} p(x, t) \\
&+s_{f}(x) p(x, t) q(x, t) \\
& \times\{1+h[1-2 p(x, t)]\} \\
&-\frac{1}{2}[p(x, t)-P(x, t)] \\
& \frac{\partial P(x, t)}{\partial t}=\frac{1_{m}^{2}}{2} \frac{\partial^{2}}{\partial x^{2}} P(x, t)+2 s_{m}(x) p(x, t) q(x, t) \\
&+[p(x, t)-P(x, t)] .
\end{aligned}
$$

The equilibrium pattern of gene frequencies is found by setting $\partial p / \partial t=0$ and $\partial P / \partial t=0$ to obtain two ordinary differential equations for the equilibria $p(x)$ and $P(x)$,

$$
\begin{gathered}
\frac{1_{f}^{2}}{2} \frac{d^{2} p}{d x^{2}}+s_{f}(x) p q\{1+h(1-2 p)\}-\frac{1}{2}(p-P)=0 \\
\frac{1_{m}^{2}}{2} \frac{d^{2} P}{d x^{2}}+2 s_{m}(x) p q+(p-P)=0 .
\end{gathered}
$$

\section{Step cline model}

In this case the selection regime takes the form shown in fig. 1 where the fitnesses of the genotypes reverse abruptly at a boundary set at $x=0$. Thus there are two pairs of equations that describe the cline, one pair for $x>0$ and the other for $x<0$. $\boldsymbol{x}>\mathbf{0}$

$$
\begin{aligned}
& \frac{d^{2} p}{d x^{2}}=\frac{-2 s_{f}}{1_{f}^{2}} p q\{1+h(1-2 p)\}+\frac{1}{1_{f}^{2}}(p-P) \\
& \frac{d^{2} P}{d x^{2}}=\frac{-4 s_{m}}{1_{m}^{2}} p q-\frac{2}{1_{m}^{2}}(p-P)
\end{aligned}
$$

$\boldsymbol{x}<\mathbf{0}$

$$
\left.\begin{array}{l}
\frac{d^{2} p}{d x^{2}}=\frac{2 s_{f}}{1_{f}^{2}} p q\{1+h(1-2 p)\}-\frac{1}{1_{f}^{2}}(p-P) \\
\frac{d^{2} P}{d x^{2}}=\frac{4 s_{m}}{1_{m}^{2}} p q+\frac{2}{1_{m}^{2}}(p-P) .
\end{array}\right\}
$$

An analytical solution to these equations cannot be obtained for the general case, instead some special cases will be examined and these reveal the major features of haplodiploid clines.

\section{Haplodiploid clines identical to diploid clines.}

Haplodiploid clines will be identical to those at corresponding diploid loci only when certain conditions are met. In general three rather restrictive

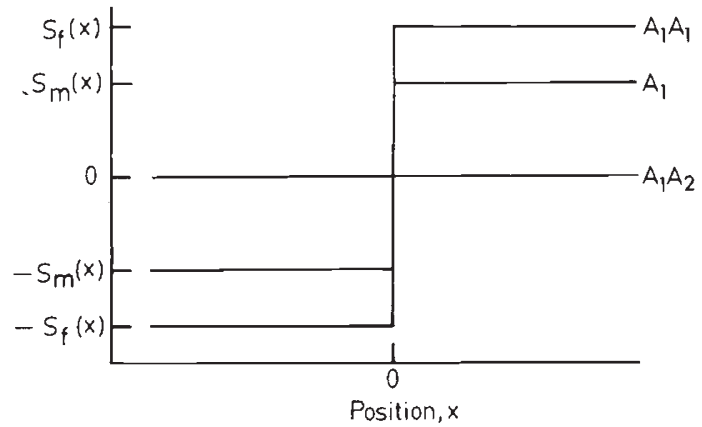

Figure 1 A step cline selection function for X-linked or haplodiploid loci. In this example the selection intensity on the males and females differs and there is no dominance in females.

conditions are required: (a) no dominance in females $(h=0)(b)$ dispersal the same in each sex $\left(1_{f}=1_{m}=1\right)(\mathrm{c}) s_{f}=2 s_{m}=s$. Substituting these into equation (11) gives

$$
\begin{aligned}
& \frac{d^{2} p}{d x^{2}}=\frac{-2 s}{1^{2}} p q+\frac{1}{1^{2}}(p-P) \\
& \frac{d^{2} P}{d x^{2}}=\frac{-2 s}{1^{2}} p q-\frac{2}{1^{2}}(p-P) .
\end{aligned}
$$

Here only $x>0$ need be considered because the cline is symmetrical around the boundary at $x=0$. Initially the gene frequencies may differ between the sexes $(P \neq p)$, but this difference will rapidly decrease tending as $t \rightarrow \infty$ to the limiting case of equality $p=P$ for all values of $x$. To see this, suppose that initially at time $t=0$ the gene frequencies are given as functions of $x$, by $p=p_{0}(x), P=$ $P_{0}(x)$. Setting $1_{f}^{2}=l_{m}^{2}=1^{2}, s_{f}=2 s_{m}=s$ and $h=0$ in the partial differential equations (7) and (8) we obtain

$$
\begin{aligned}
& \frac{\partial p}{\partial t}=\frac{1^{2}}{2} \frac{\partial^{2} p}{\partial x^{2}}-s p q-\frac{1}{2}(p-P) \\
& \frac{\partial P}{\partial t}=\frac{1^{2}}{2} \frac{\partial^{2} P}{\partial x^{2}}-s p q+(p-P) .
\end{aligned}
$$

Subtracting, we get,

$$
\frac{\partial}{\partial t}(p-P)=\frac{1^{2}}{2} \frac{\partial^{2}}{\partial x^{2}}(p-P)-\frac{3}{2}(p-P)
$$

which is the same as

$$
\frac{\partial}{\partial t}(p-P)+\frac{3}{2}(p-P)=\frac{1^{2}}{2} \frac{\partial^{2}}{\partial x^{2}}(p-P)
$$

Multiplying by the integrating factor $e^{3 t / 2}$ we obtain

$$
\frac{\partial F(x, t)}{\partial t}=\frac{1^{2}}{2} \frac{\partial^{2} F(x, t)}{\partial x^{2}},
$$


where $F(x, t)=e^{3 t / 2}(p-P)$. Now equation (15) represents a diffusion process and therefore has a solution $F=G(x, t)$ which is bounded for all $x$ and $t$ and which initially satisfies $G(x, 0)=$ $F(x, 0)=p_{0}(x)-P_{0}(x)$. Thus at any time $t>0$, we have

$$
e^{3 t / 2}(p-P)=G(x, t)
$$

therefore

$$
p-P=e^{-3 t / 2} G(x, t) \rightarrow 0 \text { as } t \rightarrow \infty,
$$

because $G(x, t)$ is bounded. Also at time $t=0$, $p-P=p_{0}(x)-P_{0}(x)$, correctly.

Therefore for the steady state the last terms of equations (13) and (14) disappear, leaving identical equations for the male and female gene frequencies in the established cline. These are also identical to the equation for a step cline at an autosomal locus with no dominance which has a well-known solution (Slatkin, 1973; Nagylaki, 1976).

The condition $s_{f}=2 s_{m}$ is worth examining in more detail as it implies that the average effect of the alleles must be the same in the males and the females, i.e., $\alpha=\beta$. With no dominance $w_{11}=1+s_{f}$, $w_{12}=1, \quad w_{22}=1-s_{f}, \quad v_{1}=1+s_{m}, \quad v_{2}=1-s_{m}$, therefore

$$
\alpha=p\left(w_{11}-w_{12}\right)+q\left(w_{12}-w_{22}\right)=s_{f}(p+q)=s_{f},
$$

and

$$
\beta=v_{1}-v_{2}=2 s_{m}
$$

Note that this condition is not the same as to what is sometimes referred to as "dosage compensation" at the level of fitness (Hartl, 1972), i.e., $w_{11}=v_{1}$, $w_{22}=v_{2}$ which would imply $s_{f}=s_{m}$.

Unless the three conditions given above are met we expect the gene frequencies in the males and females to be different. In order to gain some insight into the relative importance of these conditions the special case of no dispersal by males will be examined as this allows the equations to be put into a form to which a solution already exists. The other conditions will be relaxed in turn in order to assess their relative effects.

\section{No dispersal of males}

Consider first, in general terms, the effect of unequal dispersal of males and females when the conditions of no dominance in females and $s_{f}=2 s_{m}$ are retained. Let the males, say, have a smaller dispersal distance each generation than the females, and hence a lower rate of flow of alleles across the boundary where the selective advantage of the alternate alleles switches (fig. 1). There will be two consequences. First, the frequency of the favoured allele will be higher in males than in females. This is because after selection occurs there will be less "dilution" of the favoured allele in males due to their reduced gene flow compared to females. Second, after mating this increased allele frequency in males will be passed on to their female offspring thus steepening the female cline somewhat.

Therefore the qualitative effects of unequal dispersal alone are to cause higher allele frequencies in the sex with the lower dispersal rate and a steepening of the cline in the other sex. To investigate the quantitative effects the limiting case of no dispersal in male is now analysed.

This condition, which in fact occurs in some hymenopterans (e.g., the parasitoid Nasonia vitripennis has flightless males), allows the effects of different dispersal alone to be examined first and then the other conditions to be relaxed in turn.

If there is no dispersal of males $1_{m}^{2}=0$ and equation (10) becomes,

$$
2 s_{m}(x) p q+(p-P)=0
$$

thus

$$
(p-P)=-2 s_{m}(x) p q
$$

substituting this into equation (9) gives,

$$
\frac{1_{f}^{2}}{2} \frac{d^{2} p}{d x^{2}}+s_{f}(x) p q\{1+h(1-2 p)\}+s_{m}(x) p q=0 .
$$

No Dominance-with no dominance $h=0$, therefore, from equation (17),

$$
\frac{1_{f}^{2}}{2} \frac{d^{2} p}{d x^{2}}+\left[s_{f}(x)+s_{m}(x)\right] p q=0 .
$$

The solution to a differential equation of this form is well known (Roughgarden, 1979) and gives the female gene frequency:

$x>0$

$$
p(x)=-\frac{1}{2}+\left(\frac{3}{2}\right)\left[\tanh \left(\frac{\sqrt{s_{f}+s_{m}}}{1_{f} \sqrt{2}} x+c\right)\right]
$$

$x<0$

$$
p(x)=\frac{3}{2}-\left(\frac{3}{2}\right)\left[\tanh \left(-\frac{\sqrt{\mathrm{s}_{\mathrm{f}}+\mathrm{s}_{\mathrm{m}}}}{1_{\mathrm{f}} \sqrt{2}} \mathrm{x}+\mathrm{c}\right)\right]^{2}
$$

where $c=\operatorname{arctanh}((\sqrt{2 / 3})$. 
The male gene frequencies, from equation (16), are

$x>0$

$$
P(x)=2 s_{m} p(x) q(x)+p(x)
$$

$x<0$

$$
P(x)=p(x)-2 s_{m} p(x) q(x) .
$$

The effect of different dispersal alone can be seen if condition (c), $s_{f}=2 s_{m}$ is retained. An example is shown in fig. 2. Although gene frequencies in the sexes do differ as a result of unequal dispersal the difference is very small, and probably would be undetectable in a natural population with realistic sample sizes. If the condition is relaxed then larger gene frequency differences do occur (fig. 2), however moderately strong selection and a considerable difference between the male and female selection coefficients are required. Note that in the example shown in fig. 2(b) although selection is relatively strong $\left(s_{f}=0.01, s_{m}=0.1\right)$ the accuracy of the solution is good. The original selection equations (3) and (4) are quite accurate with

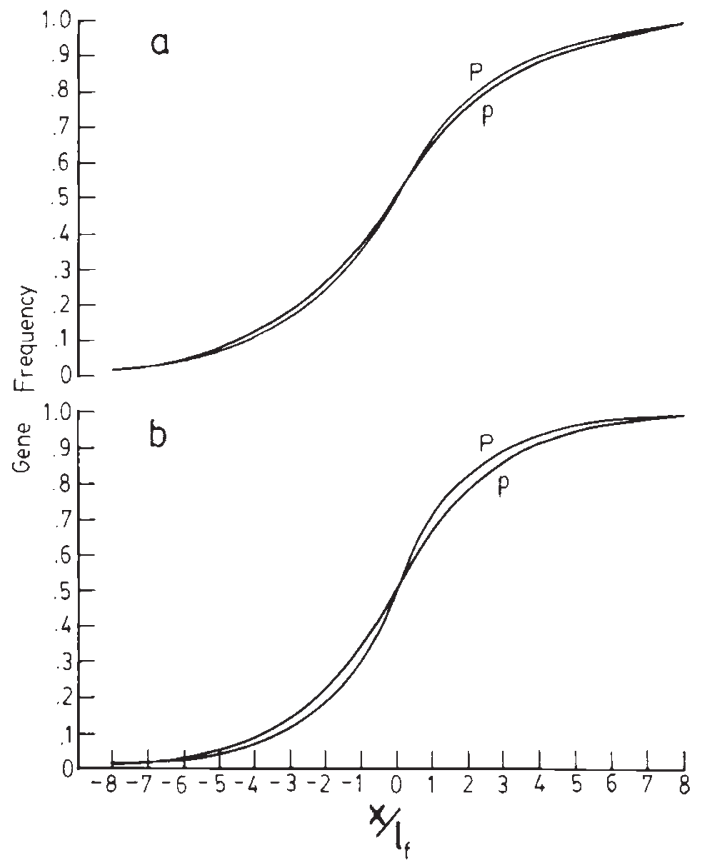

Figure 2 Gene frequency clines in haplodiploids when there is no dispersal by males $\left(1_{m}^{2}=0\right)$ and no dominance in females. The examples were calculated using equations (19)-(22). (a) If the average effect of alleles is the same in males and females $\left(s_{f}=2 s_{m}=0.05\right)$ then only small gene frequency differences occur. (b) An appreciable gene frequency difference exists only when the selection intensity on each sex is considerably different $\left(s_{f}=0.01\right.$, $\left.s_{m}=0 \cdot 1\right)$. this intensity of selection (see Appendix) as is the solution to the diffusion equation (Slatkin, 1973; Roughgarden, 1979).

Dominance-consider the $A_{1}$ allele, at frequencies $(p, P)$, to have a degree of dominance over the recessive allele $A_{2}$, at frequencies $(q, Q)$ i.e., $h>0$. Equation (17) can be rewritten as,

$$
\frac{1_{f}^{2}}{2} \frac{d^{2} p}{d x^{2}}+\left[s_{f}(x)+s_{m}(x)\right] p q+s_{f}(x) p q h(1-2 p)=0
$$

which is equivalent to

$$
\frac{1_{f}^{2}}{2} \frac{d^{2} p}{d x^{2}}+\left[s_{f}(x)+s_{m}(x)\right] p q\left\{1+h^{*}(1-2 p)\right\}=0
$$

where the substitution

$$
h^{*}=h s_{f}(x) /\left(s_{f}(x)+s_{m}(x)\right) .
$$

Let $s(x)=s_{f}(x)+s_{m}(x)$ and $h^{*}=1$ then equation (24) becomes,

$$
\frac{1_{f}^{2}}{2} \frac{d^{2} p}{d x^{2}}+2 s(x) p^{2} q=0 .
$$

The equivalent equations for the recessive $A_{2}$ allele are. For $x>0$,

$$
\frac{1_{f}^{2}}{2} \frac{d^{2} q}{d x^{2}}+2 s p q^{2}=0
$$

and for $x<0$,

$$
\frac{1_{f}^{2}}{2} \frac{d^{2} q}{d x^{2}}-2 s p q^{2}=0 .
$$

Also

$$
Q=2 s_{m}(x) p q+q .
$$

Equations (26) and (27) are the same form as the equations for a step cline at an autosomal locus with dominance, therefore the solution to these given by Haldane (1948) can be used directly to obtain the female gene frequencies. Note that since $h^{*}=1$, the actual degree of dominance implied by equation (24), given by equation (25), $h=$ $\left[s_{m}(x)+s_{f}(x)\right] / s_{f}(x)$. Unless $s_{m}(x)=0$ then the case modelled by these equations represents some overdominance for $x>0$ and underdominance for $x<0$. In Haldane's model it is the recessive allele which is favoured for $x>0$ and the dominant allele favoured for $x<0$, hence for consistency the selective values are now

$$
\begin{array}{ll}
w_{11}=1-s_{f}(x) & v_{1}=1-s_{m}(x) \\
w_{12}=1-h s_{f}(x) & \\
w_{22}=1+s_{f}(x) & v_{2}=1+s_{m}(x) .
\end{array}
$$


The solutions to equations (26) and (27) given by Haldane (1948) are;

$\boldsymbol{x}>\mathbf{0}$

$$
\begin{aligned}
2 \sqrt{s} \frac{x}{1_{f}}= & \sinh ^{-1}\left[\frac{\sqrt{2}(1+2 q(x))}{1-q(x)}\right] \\
& -\sinh ^{-1}\left[\frac{\sqrt{2}(1+2 b)}{1-b}\right]
\end{aligned}
$$

$x<0$

$$
q(x)=\frac{1_{f}^{2} b}{1_{f}^{2}-x 1_{f} \sqrt{[2 s(4-3 b) / 3]}+2 b s x^{2} / 3},
$$

where $b=0.6143$ because in this case the intensity of selection is assumed to be equal on each side of the boundary (Haldane 1948).

The corresponding male gene frequencies, from equation (28) are,

$$
\boldsymbol{x}>\mathbf{0}
$$

$$
Q(x)=2 s_{m}(x) p(x) q(x)+q(x)
$$

$x<0$

$$
Q(x)=q(x)-2 s_{m}(x) p(x) q(x) .
$$

The effect of relaxing all three of the original conditions (a), (b) and (c) can now be examined.
Figure 3 shows an example of a gene frequency cline in haplodiploids with different dispersal in each sex $\left(1_{m}^{2}=0\right)$, dominance in females and unequal average effect of alleles in each sex $\left(s_{f} \neq\right.$ $2 s_{m}$ ). In this example with $s_{f}=0.01, s_{m}=0.05$ the viabilities are:

\begin{tabular}{cccccc} 
& $w_{11}$ & $w_{12}$ & $w_{22}$ & $v_{1}$ & $v_{2}$ \\
\cline { 2 - 6 }$x>0$ & 0.99 & 0.94 & 1.01 & 0.95 & 1.05 \\
$x<0$ & 1.01 & 1.06 & 0.99 & 1.05 & 0.95
\end{tabular}

hence there is also some heterozygous advantage and disadvantage. The effect of dominance in females is to alter the shape of the cline. However, unless there is a considerable difference in the intensity of selection acting on the males and females then an appreciable gene frequency difference will not occur.

In summary then, if any of the three conditions (a) no dominance in females (b) equal dispersal distance by each sex (c) equal average effect of alleles in males and females, are not met then a gene frequency difference between the sexes will be established. The values of the selection coefficients $s_{f}$ and $s_{m}$ have the greatest effect on the size of the gene frequency difference. However even with different selection intensities the male

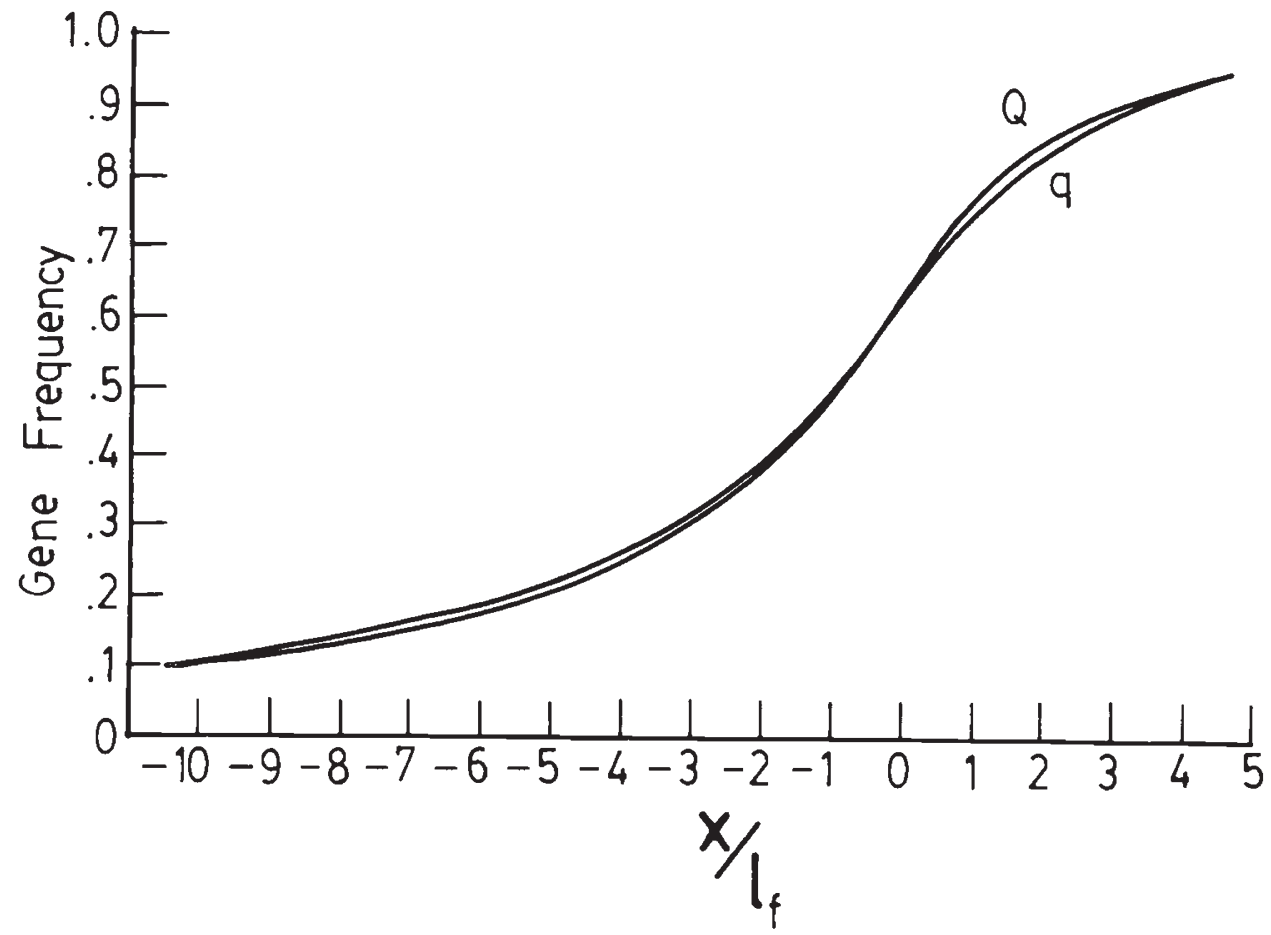

Figure 3 An example of a gene frequency cline in haplodiploids when there is no dispersal by males $\left(1_{m}^{2}=0\right)$, dominance in females and unequal average effect of alleles in each $\operatorname{sex}\left(s_{f}=0.01, s_{m}=0.05\right)$. The cline was calculated using equations (29)-(32). 
and female gene frequencies are likely to be very close. Conversely even quite large differences in selection intensity may not be translated into gene frequency differences large enough to be detected in natural populations.

[It was pointed out by a reviewer that even if all three conditions were violated then the male and female gene frequencies could be identical provided,

$$
21_{f}^{2} s_{m}=s_{f} 1_{m}^{2}[1+h(1-2 p)] .
$$

The biologically possible case that satisfies this is when $h=0$ and $s_{m} / s_{f}=1_{m}^{2} / 21_{f}^{2}$. The form of the cline is given by the ordinary diploid differential equation (Nagylaki, 1976)].

\section{Ploidy-weighted gene frequency}

I am indebted to an anonymous reviewer for drawing attention to the interest attaching to $\bar{p}$ defined as $(2 p+P) / 3$, which can be regarded as the gene frequency averaged over the two sexes with weights proportional to the ploidys. Thus

$$
p=\bar{p}+(p-P) / 3, \quad P=\bar{p}-2(p-P) / 3
$$

and $q=\bar{p}-(p-P) / 3$, from which it follows that

$$
\begin{aligned}
\frac{\partial p}{\partial t}= & \frac{1_{f}^{2}}{2} \frac{\partial^{2} \bar{p}}{\partial x^{2}}+\frac{1_{f}^{2}}{6} \frac{\partial^{2}}{\partial x^{2}}(p-P)-\frac{1}{2}(p-P) \\
& +s_{f} \bar{p} \bar{q}[1+h(1-2 \bar{p})]+0[s(p-P)], \\
\frac{\partial P}{\partial t}= & \frac{1_{m}^{2}}{2} \frac{\partial^{2} \bar{p}}{\partial x^{2}}-\frac{1_{m}^{2}}{3} \frac{\partial^{2}}{\partial x^{2}}(p-P)+(p-P) \\
& +s_{m} \bar{p} \bar{q}+0[s(p-P)],
\end{aligned}
$$

where $s$ is the larger of the maximum values of $s_{f}(x)$ and $s_{m}(x)$ for all values of $x$, and $0[y]$, for any variable $y$, denotes a quantity of order of magnitude not greater than $y$.

Consequently

$$
\begin{aligned}
\frac{\partial \bar{p}}{\partial t}= & \frac{2}{3} \frac{\partial p}{\partial t}+\frac{1}{3} \frac{\partial P}{\partial t} \\
= & \frac{21_{f}^{2}+1_{m}^{2}}{6} \frac{\partial^{2} \bar{p}}{\partial x^{2}}+\bar{s} \bar{p} \bar{q} \\
& +\frac{1_{f}^{2}-1_{m}^{2}}{9} \frac{\partial^{2}(p-P)}{\partial x^{2}}+0[s(p-P)],
\end{aligned}
$$

where $\bar{s}=(2 / 3) s_{f}[1+h(1-2 \bar{p})]+s_{m} / 3$.

To begin with consider the special case in which male and female dispersions are equal, i.e., $1_{f}=$ $1_{m}=1$, but no other special assumptions are made.
Then

$$
\frac{\partial \bar{p}}{\partial t}=\frac{1^{2}}{2} \frac{\partial^{2} \bar{p}}{\partial x^{2}}+\bar{s} \bar{p} \bar{q}+0[s(p-P)] .
$$

Also

$$
\begin{aligned}
\frac{\partial(p-P)}{\partial t}= & \frac{1^{2}}{2} \frac{\partial^{2}(p-P)}{\partial x^{2}}-\frac{3}{2}(p-P) \\
& +\left\{s_{f} p q[1+h(1-2 p)]-s_{m} p q\right\}, \\
= & \frac{1^{2}}{2} \frac{\partial^{2}(p-P)}{\partial x^{2}}-\frac{3}{2}(p-P)+0(s) .
\end{aligned}
$$

In the steady state $(p-P) / t=0$ and $(p-P)$ satisfies

$$
\left(\frac{1^{2}}{2} \frac{d^{2}}{d x^{2}}-\frac{3}{2}\right)(p-P)=0(s) .
$$

Proceeding heuristicaly, without attempting a mathematically rigorous argument, we might reasonably assume that $(p-P)=0(s)$. It follows that when the cline is established, then

$$
\frac{1^{2}}{2} \frac{\partial^{2} \bar{p}}{\partial x^{2}}+\bar{s} \bar{p} \bar{q}=0[s(p-P)]=0\left(s^{2}\right),
$$

and by heuristic reasoning we might infer with some degree of plausibility that the cline in $\bar{p}=$ $(2 p+P) / 3$ approximates, with proportionate error of order $s$ only, to that of the corresponding diploid cline with mobilities 1 and selection coefficients $s_{f}(x)$ and $s_{m}(x)$, presumed small.

The foregoing admits of a slight extension. If $1_{f}$ and $1_{m}$ are equal with a proportionate error $0(s)$ the same conclusion follows provided that $\left(1_{f}^{2}-\right.$ $\left.1_{m}^{2}\right)=0(s)$.

It would be possible to go even further if the following argument proposed by the reviewer is mathematically valid. Make a "stretching transformation", $x=\xi / s$. That is we rescale the measurement of distance, so that distance is described by the new variable $\xi$ which is $x \sqrt{s}$ (Slatkin, 1973). Then in the steady state

$$
\begin{aligned}
& \frac{1^{2}}{2} s \frac{\partial^{2} \bar{p}}{\partial \xi^{2}}+\bar{s} \bar{p} \bar{q}+\frac{\left(1_{f}^{2}-1^{2} m\right)}{9} s \frac{\partial^{2}}{\partial \xi^{2}}(p-P) \\
& \quad+0[s(p-P)]=0 .
\end{aligned}
$$

Assuming, as before, that $(p-P)=0(s)$, this is

$$
s\left[\frac{1^{2}}{2} \frac{\partial^{2} \bar{p}}{\partial \xi^{2}}+\left(\frac{\bar{s}}{s}\right) \bar{p} \bar{q}\right]+0\left(s^{2}\right)+0\left(s^{2}\right)=0 .
$$

Dividing through by $s$ gives

$$
\frac{1^{2}}{2} \frac{\partial^{2} \bar{p}}{\partial \xi^{2}}+\left(\frac{\bar{s}}{s}\right) \bar{p} \bar{q}+0(s)=0 .
$$


The first two terms are (formally, at least) of magnitude $0(1)$. Thus heuristically we would obtain the result that with proportionate error $0(s)$, the ploidy-weighted average gene frequency $\bar{p}$ exhibits a cline.

$$
\bar{p}=F(\xi, 1, \bar{s} / s)=F(x \sqrt{s}, 1, \bar{s} / s)
$$

where $F(x, 1, \bar{s} / s)$ is the diploid cline with dispersion 1 and selection coefficient $\bar{s} / s$.

\section{GENE FREQUENCY CLINE IN BOMBUS MELANOPYGUS}

Relatively few gene frequency clines are known at $\mathrm{X}$-linked or haplodiploid loci, however a good example is provided by the cline in colour morph frequencies in the bumble bee $B$. melanopygus Nylander. This species exhibits a red/black colour dimorphism of the pile on abdominal terga 2 and 3 , which is controlled by a single diallelic locus with the red $(R)$ allele dominant to the black $(r)$ allele (Owen and Plowright 1980). The gene frequencies form a cline down the west coast of North America. The red allele is near fixation in Washington state and northern Oregon, but to the south it is progressively replaced by the black allele which reaches fixation in northern California. Exact gene frequency estimates are available from only four locations along the coast of Oregon and California (Owen and Plowright, 1980; Owen, 1982), and these are shown with their 95 per cent confidence limits in fig. 4. However additional collection records from Stephen (1957) confirm that these estimates do in fact give a true picture of the cline (fig. 5). Nevertheless one complicating factor is that prior to Owen and Plowright's (1980) work the red and black morphs were assumed to represent different species; $B$. melanopygus and $B$. edwardsii respectively. This now appears improbable given the finding of Mendelian segregation of the two colour morphs in colonies raised from queens caught at Cape Perpetua, and Crescent City (fig. 4), and that the queen genotype frequencies were not significantly different from those expected under Hardy-Weinberg equilibrium (Owen and Plowright, 1980). Moreover the morphological characters used to differentiate the queens of the nominate "species" (Stephen, 1957) differ only quantitatively not qualitatively, showing variation that is quite consistent with that expected in a single species with an extensive geographical range (Owen, 1982). The possibility that the cline is in fact caused by hybridization between "true" melanopygus and edwardsii, or results from secondary intergradation cannot, of course, be discounted. However the simplest interpretation, based on the genetic evidence, is of a single species exhibiting clinal variation in morph frequencies (Owen and Plowright, 1980) and for the purposes of this paper will be treated as such.

Haldane (1948) remarked that "in certain cases a cline can give quantitative information regarding the intensity of natural selection". In this section an approximate estimate of the likely selection intensity acting on the $B$. melanopygus is obtained by applying haplodiploid cline theory.

Direct gene frequency estimates are available for queens only (fig. 4) and not for males, however it appears that the gene frequencies are not sig-

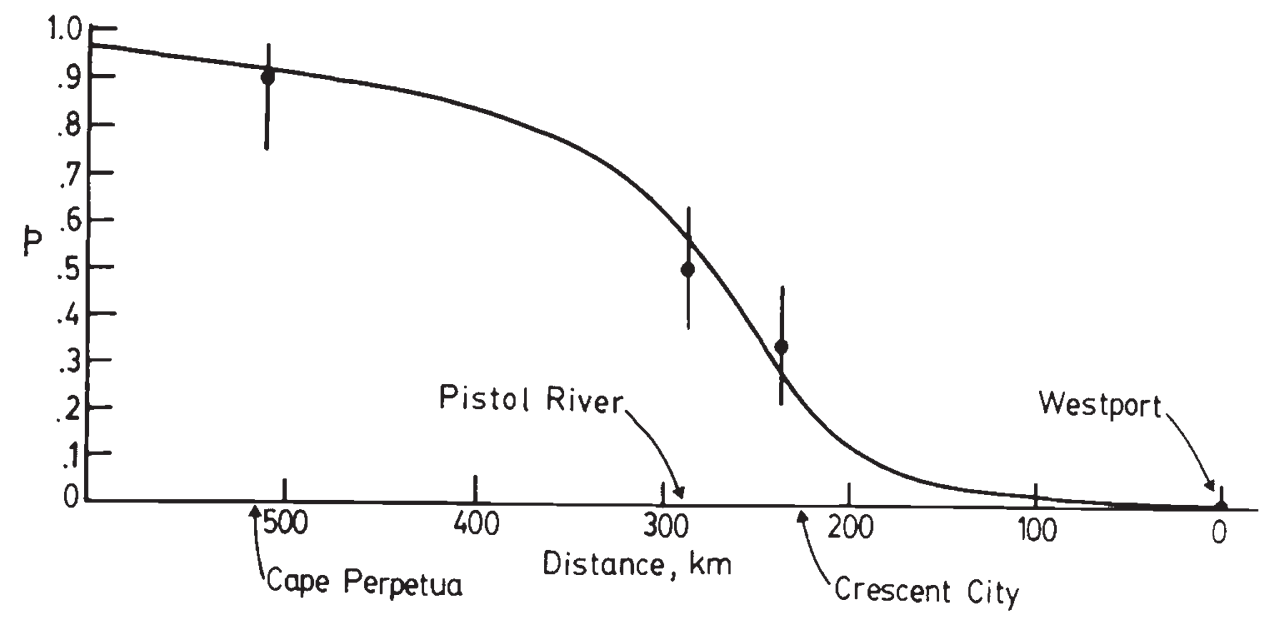

Figure 4 Frequency $(p)$ of the dominant red $(R)$ allele ( \pm 95 per cent confidence limits) in queens of the bumble bee Bombus melanopygus along the coasts of Oregon and California. Also shown is the theoretical cline calculated using equations (29) and (30), see text and table 1 for details. 


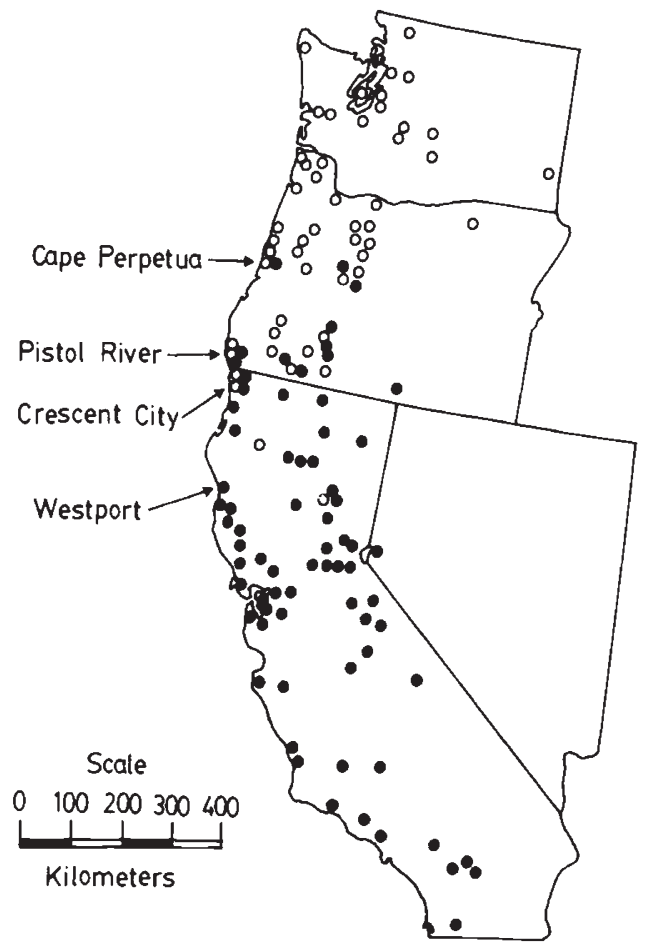

Figure 5 Distributions of the red $(O)$ and black $(O)$ morphs of Bombus melanopygus, based on collection data given by Stephen (1957) and Owen and Plowright (1980). The two morphs previously were assumed to represent different species, B. melanopygus and B. edwardsii respectively.

nificantly different, at least not detectably so. This can be inferred from the observation that the queen genotype frequencies are in Hardy-Weinberg equilibrium and that the observed numbers of different colony type are not significantly different from those expected on the assumption of equal gene frequencies (Owen and Plowright 1980). Indeed this is what would be expected in the light of the theory developed in the preceding sections. Unless the difference in the intensity of selection aciing on males and females is very large, then any gene frequency difference will be negligible and certainly undetectable with realistic sample sizes.

If there is dominance, unequal dispersal of males and females and $s_{f} \neq 2 s_{m}$, then an analytical solution for the general case of a haplodiploid cline is not possible. The pile colour locus of $B$. melanopygus shows dominance and there is no information on the relative dispersal of males and females. Hence in order to fit a cline model to the data it is necessary to make two simplifying assumptions, (a) selection acts only on the queens (i.e., $s_{m}=0$ ). (b) there is no dispersal of males (i.e., $\left.1_{m}^{2}=0\right)$. If this is done then, because $h=$ $\left(s_{f}(x)+0\right) / s_{f}(x)=1$, equations (29) and (30) can be used to give the female allele frequencies and the male allele frequencies, given by equations (31) and (32) are identical, i.e., $Q(x)=q(x)$. The second assumption is certainly incorrect as there is undoubtedly dispersal by male bumble bees. However all this means is that the estimate of $s_{f}$ obtained is a minimum estimate. Dispersal of males will increase the level of gene flow in the system causing the cline to flatten somewhat therefore a correspondingly larger $s_{f}$ would be required to produce a cline of the same steepness.

If there is also selection on the males then the situation admittedly becomes more complicated and cannot be modelled easily. The best that can be done is to take the estimate of $s_{f}$ as a measure of the overall selection, $s$, acting on both males and females. In fact this will be quite accurate if $s$ is small because even with dominance and different dispersal rates the gene frequency difference between males and females will be very small.

There is little information regarding the dispersal of bumble bee queens. The distance over which a queen disperses from her parental nest may be influenced by factors such as the availability of forage, suitable hibernation sites and acceptable nest sites the following spring. A study by Bowers (1985) suggests that the average dispersal distance of Bombus queens between subalpine meadows is small. Using the data shown in Bower's fig. 4 I calculate the standard deviation $\left(1_{f}\right)$ of the dispersal regime to be $0.232 \mathrm{~km}$. Now the subalpine meadows occur as islands of suitable habitat in coniferous forests. In more open, homogeneous countryside the dispersal distance of queens may well be greater, however even if they disperse 20 times as far then $1_{f}=5 \mathrm{~km}$ only.

Equations (29) and (30) were used to generate the curve shown in fig. 4 . The equivalent combinations of $s_{f}$ and $1_{f}$ that produce the curve are given in table 1 and a comparison of the observed and predicted allele frequencies is given in table 2 . The theoretical cline was "anchored" at distance 0 (Westport in real life) by setting $p=0.001$ here, then the curve which best fitted, by eye, the observed data points was chosen.

The fit is moderately good although the observed cline appears to be somewhat flatter than the predicted cline over the central region (Pistol River-Crescent City). However this is what would be expected if dispersal of males does in fact occur as is undoubtedly the case. The values of $s_{f}$ given in table 1 must therefore be interpreted as minimum estimates. If $1_{f}$ is in the $5-10 \mathrm{~km}$ range 
Table 1 Combinations of $s_{f}$, the selection coefficient on queens, and $1_{f}$, the dispersal distance of queens, that give the theoretical cline in fig. 4 , calculated using equations (29) and (30). Since it is assumed that $1_{m}^{2}=0$ the values of $s_{f}$ are minimum estimates

\begin{tabular}{lr}
\hline$s_{f}$ & $1_{f}(\mathrm{~km})$ \\
\hline 0.00094 & $2 \cdot 5$ \\
0.00375 & $5 \cdot 0$ \\
0.015 & $10 \cdot 0$ \\
0.060 & $20 \cdot 0$ \\
\hline
\end{tabular}

Table 2 Comparison of the observed and predicted frequency, $p$, of the red $(R)$ allele in $B$. melanopygus queens at four locations in Oregon and California (also see figs. 4 and 5 ). The predicted value were obtained from the curve in fig. 4

\begin{tabular}{lll}
\hline & $\begin{array}{l}\text { Observed }( \pm 95 \\
\text { per cent } \\
\text { conf. limits) }\end{array}$ & Predicted \\
\hline Cocation & $\begin{array}{r}0.900+0.065 \\
-0.156\end{array}$ & 0.918 \\
Pistol River & $\begin{array}{l}0.506 \pm 0.133 \\
\text { Cresent City }\end{array}$ & 0.569 \\
Westport & $0.000+0.002$ & 0.280 \\
\hline
\end{tabular}

(which may well be an overestimate) then a minimum selection intensity on the order of 1 per cent or less is sufficient to maintain the cline.

The possible adaptive significance of this colour dimorphism has been discussed elsewhere (Owen and Plowright, 1980; Plowright and Owen, 1980 ), and it has been speculated that selection for Müllerian mimicry may favour the red morph in the north and the black one in the south thus generating the cline.

Acknowledgements I thank Dr A. R. G. Owen for his help and advice with this problem, and an anonymous reviewer for his helpful comments. This work was supported by a grant from the Natural Sciences and Engineering Research Council of Canada.

\section{REFERENCES}

BOWERS, M. A. 1985. Bumble bee colonization, extinction, and reproduction in subalpine meadows in northeastern Utah. Ecology, 66, 914-927.

ENDLER, J. A. 1977. Geographic Variation, Specification, and Clines. Princeton University Press, Princeton, $246 \mathrm{pp}$.

FISHER, R. A. 1937. The wave of advance of advantageous genes. Ann. Eugen., 7, 355-369.

FISHER, R. A. 1950. Gene frequencies in a cline determined by selection and diffusion. Biometrics, 6, 353-361.

FLEMING, W. H. 1975. A selection-migration model in population genetics. J. Math. Biol, 2, 219-233.

HALDANE, J. B. S. 1948. The theory of a cline. J. Genet., 48, 277-284.

HARTL, D. L. 1972. A fundamental theorem of natural selection for sex-linkage or arrhenotoky. Am. Natur., 106, 516-524.

JOHNSON, E. M., SCHALTER, H. E., GILLASPY, J. E. AND ROCK WOOD, E. S. 1969. Isozyme genotype-environment relationships in natural populations of the harvester ant Pogonomyrmex barbatus from Texas. Biochem. Genet., 3, 429-434.

MAY, R. M., ENDLER, J. A. AND McMURTRIE, R. E. 1975. Gene frequency clines in the presence of selection opposed by gene flow. Am. Natur., 109, 659-676.

NAGYLAKI, T. 1975. Conditions for the exitence of clines. Genetics, 80, 595-615.

NAGYLAKI, T. 1976. Clines with variable migration. Genetics, $83,867-886$.

OWEN, R. E. 1980. Population genetics of social hymenoptera with worker produced males. Heredity, 45, 31-46.

OWEN, R. E. 1982. Contributions to the study of the genetics and evolutionary biology of bumble bees (Hymenoptera: Apidae). $\mathrm{Ph} . \mathrm{D}$. thesis, University of Toronto, Toronto, Ontario, $299 \mathrm{pp}$.

OWEN, R. E. AND Plowright, R. C. 1980. Abdominal pile color dimorphism in the bumble bee, Bombus melanopygus. J. Hered., 71, 241-247.

PLOWRIGHT, R. C. AND OWEN, R. E. 1980. The evolutionary significance of bumble bee color patterns: a mimetic interpretation. Evolution, 34, 622-637.

ROUGHgARDEN, J. 1979. Theory of Population Genetics and Evolutionary Ecology: An Introduction. Macmillan Publishing Co., Inc., New York, 634 pp.

SLATKIN, M. 1973. Gene flow and selection in a cline. Genetics, $75,733-756$.

SLATKIN, M. 1975. Gene flow and selection in a two locus system. Genetics, $81,787-802$.

STEPHEN, W. P. 1957. Bumble bees of Western America (Hymenoptera: Apoidea). Oregon Agric. Exp. Stn. Tech. Bull., 40, 1-163. 


\section{APPENDIX}

The accuracy of equations (3) and (4) under moderately strong selection $\left(s_{f}=0.01, s_{m}=0 \cdot 10\right)$ was assessed by comparison with iterations of the exact recurrence equations for selection at an X-linked locus. The recurrence equations were iterated and the approximate equations evaluated for 50 generations starting from $p_{0}=P_{0}=0 \cdot 5$. The results are given in the table. It can be seen that the approximate equations are quite accurate (within $\sim 1$ per cent) even with selection of this intensity.

\begin{tabular}{|c|c|c|c|c|c|}
\hline $\begin{array}{l}\text { Generation } \\
\text { Exact }\end{array}$ & $p$ & $\Delta p p$ & $\Delta P$ & $(p-P)$ & \\
\hline 5 & 0.59834 & 0.01819 & 0.62810 & 0.01636 & -0.02976 \\
\hline 10 & 0.68212 & 0.01603 & 0.70915 & 0.01532 & -0.02703 \\
\hline 20 & 0.81634 & 0.01115 & 0.83476 & $0 \cdot 01027$ & $-0 \cdot 01842$ \\
\hline 30 & 0.90176 & 0.00661 & 0.91255 & 0.00596 & -0.01079 \\
\hline 40 & 0.94981 & 0.00356 & 0.95559 & 0.00317 & -0.00578 \\
\hline 50 & 0.97498 & 0.00182 & 0.97793 & 0.00161 & -0.00295 \\
\hline \multicolumn{6}{|l|}{ Approximate } \\
\hline 5 & 0.59895 & 0.01845 & 0.62920 & 0.01667 & -0.03025 \\
\hline 10 & 0.68463 & $0 \cdot 01648$ & $0 \cdot 71249$ & 0.01582 & -0.02786 \\
\hline 20 & 0.82338 & $0 \cdot 01151$ & 0.84242 & 0.01058 & -0.01904 \\
\hline 30 & 0.91044 & 0.00661 & 0.92122 & 0.00591 & -0.01078 \\
\hline 40 & 0.95723 & 0.00336 & 0.96267 & 0.00296 & -0.00544 \\
\hline 50 & 0.98020 & 0.00160 & 0.92879 & 0.00140 & -0.00259 \\
\hline
\end{tabular}

